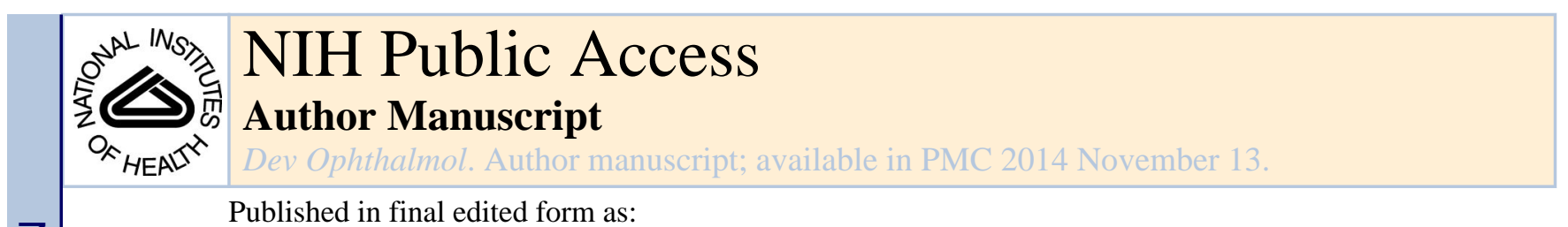

Published in final edited form as:

Dev Ophthalmol. 2014 ; 53: 167-177. doi:10.1159/000357376.

\title{
CELL AND GENE THERAPY
}

Rajesh C. Rao, MD and

W.K. Kellogg Eye Center, University of Michigan Medical School, 1000 Wall St., Ann Arbor, MI 48105 USA

David N. Zacks, MD

W.K. Kellogg Eye Center, University of Michigan Medical School, 1000 Wall St., Ann Arbor, MI 48105 USA

David N. Zacks: davzacks@umich.edu

\section{ABSTRACT/INTRODUCTION}

Gene therapy, or vector-mediated delivery of specific genes to retinal cells deficient in these genes, has emerged as a promising clinical strategy for retinal degenerative disease, such as Leber's congenital amaurosis (LCA) associated with RPE65 mutations. ${ }^{1,2}$ However, recent reports indicate that despite a improvement in visual function, RPE65-LCA-associated retinal degeneration continues unabated. ${ }^{3}$ These findings highlight the fact that gene augmentation therapy to restore the missing chromophore does not limit the progressive death of photoreceptors and other retinal cells. Therefore, in hereditary forms of retinal degenerations associated with known gene mutations, two factors need to be addressed: replacement of the missing gene and cell survival. Combining cell and gene therapy may offer a therapeutic avenue that allows both problems to be addressed.

\section{CELL THERAPY: GRAFTING WILD-TYPE, ALLOGENEIC PHOTORECEPTORS AND RETINAL PIGMENT EPITHELIUM TO RESCUE GENETIC DEFECTS}

The vitreous and subretinal spaces are generally considered to be an immune-privileged site, allowing extended or indefinite survival of allogeneic grafts. ${ }^{4}$ Due to immune-privilege it should be possible to transplant allogeneic retinal cells, such as wild-type photoreceptors or retinal pigment epithelium (RPE) ${ }^{5}$ which carry wild-type alleles of missing genes, such as $R P E 65$, in order to replace missing gene products and degenerating retinal cells in critical areas such as the macula, without immune rejection. The transplanted cells may be relatively resistant to degeneration because they carry the wild-type alleles of the missing gene. A recent study in the zebrafish cone phosphodiesterase mutant pde $6 c^{\mathrm{w} 59}$, in which cones die soon after they are formed, subsequently followed by rod loss in the central retina, offered evidence that wild-type cell transplants provide the wild-type allele and remain viable, even if neighboring pde $6 \mathrm{c}^{\mathrm{w} 59}$ photoreceptors die. In this case, wild type cones were transplanted in the pde $6 c^{\text {w59 }}$ retina, creating a mosaic. Mutant cones in the mosaic retina continued to die at similar rates compared to non-mosaic, purely mutant retina. Interestingly, in mosaic retinae-similar to purely mutant retinae-mutant pde $6 \mathrm{c}^{\mathrm{w} 59}$ cone death continued to exert a "bystander effect" on mutant pde $6 c^{\mathrm{w} 59}$ rods, i.e. mutant cone death induced subsequent 
mutant rod loss. However, transplanted wild-type cones showed little or no death. These data showed two critical features of transplanted wild-type cone cells: 1) grafted cells replace the missing enzyme, and 2) integrated cells display persistent survival even while surrounding mutant photoreceptors continue to die. ${ }^{6}$

In specific retinal dystrophies, a gene mutation causes dysfunction in the photoreceptor, but does not cause structural retinal degeneration. These disorders include a variety of rod and cone dystrophies such as congenital stationary night blindness (CSNB), red-green color blindness, monochromatism, and achromatopsia. While these diseases may be suitable candidates for gene therapy, cell therapy to deliver the missing gene via transplant wild-type differentiated photoreceptor-containing the normal copy of the deficient gene-may also offer a therapeutic avenue. A recent study demonstrated that transplantation of donor photoreceptors, which express green fluorescent protein (GFP) under control of the promoter of the rod specific transcription factor $\mathrm{Nrl}$, into adult $\mathrm{Gnat}^{-/}{ }^{-}$retina, a model for CSNB, resulted in integration leading to partial restoration of vision. ${ }^{7}$ In this case 200,000 fluorescence-activated cell sorted (FACS) $\mathrm{Nrl}$-GFP+ post-mitotic rod precursors were transplanted into the subretinal space of adult Gnat $^{-1-}$ mutant retina. About $15 \%$ of the $\mathrm{Nrl}$-GFP+ rods integration into the outer nuclear layer of the mutant retina and distributed over more than $50 \%$ of the retina. Because the nnat $^{-/-}$mutant retina lacks alphatransducin (Gnat1), a protein required for rod phototransduction, non-transplanted mice do not have functional rods and thus the mice do not respond to scotopic stimuli. The mutant mice do have normal cone structure and do respond to photopic stimuli. In contrast to untreated or sham-treated $\mathrm{Gnatl}^{-/-}$mice, transplanted $\mathrm{Gnatl}^{-/-}$mice showed optomotor head-tracking, improved performance in visually guided water-maze test under scotopic conditions, and positive visual stimuli-evoked signals in the visual cortex. Interestingly, there was no transplant-associated change in the electroretinogram (ERG). Taken together, this promising cell therapy study demonstrated restoration of visual function in a CSNB mouse model—a stationary disease with little photoreceptor death—using transplanted $\mathrm{Gnat}^{+/+}$(wild-type), Nrl-GFP+ post-mitotic rod precursors.

The degree of photoreceptor maturation and integration depends on disease model and degeneration kinetics. These factors thus impact the ability of wild type cell transplants to correct the underlying genetic etiology. Two important studies highlight these aspects.

In the first report, FACS-sorted photoreceptors were isolated from $\mathrm{Cr} x$-GFP transgenic mouse, and transplanted into wild-type and mutant retinae characterized by two mutations associated with Leber's congenital amaurosis, $\mathrm{Crb}^{r d 8 / r d 8}$ and $\mathrm{Gucy} 2 e^{-/-} .8 \mathrm{Crx}$ is a transcription factor expressed by postmitotic rod and cone precursors as well as differentiated photoreceptors. In the murine retina, cones are born prenatally, while rods emerge postnatally. The investigators found that subretinal transplantation of postnatal versus embryonic Crx-GFP donor cells resulted in a 10-fold higher integration of rods. Moreover, transplantation of embryonic $\mathrm{Cr} x$-GFP precursors in the relatively cone deficient Gucy $2 e^{-/-}$mutant retina resulted in a higher proportion of differentiation cones among the integrated donor cells than in the more cone rich wild type and $\mathrm{Crbl}^{r d 8 / r d 8}$ retinae. This work demonstrated that host cone depletion is more permissive for donor $\mathrm{Cr} x$-GFP 
precursor cone differentiation, and the $\mathrm{Crx}$ cell therapy allows for cone replacement in the adult retina.

A recent study reported efficiencies of $\mathrm{Nrl}-\mathrm{GFP}+$ rod integration across a variety of murine genetic backgrounds, with varying levels of outer nuclear layer degeneration, including

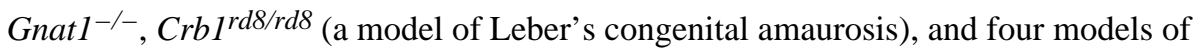
retinitis pigmentosa (RP): $P r p h 2^{+/ \Delta 307}, \operatorname{Prph} 2^{r d 2 / r d 2}, R h o^{-/-}, P D E 6 \beta^{r d 1 / r d 1}{ }^{9}{ }^{9}$ Each model is associated with loss of rods over a period ranging from $\sim 10 \%$ loss over 12 months $\left(\right.$ Gnat $\left.^{-/-}\right)$to near complete loss of rods within 3 weeks $\left(P D E 6 \beta^{r d l / r d l}\right)$. Three weeks after subretinal transplantation of $\mathrm{Nrl}$-GFP+ rod precursors into 6 to 8 week-old recipients, integration to the outer nuclear layer was highest in the $\mathrm{Crb}^{\mathrm{rd} d 8 / \mathrm{rd} 8}$ strain. Integration to the outer nuclear layer in wild-type, $\operatorname{Prph} 2^{+/ \Delta 307}, \operatorname{Prph} 2^{r d 2 / r d 2}, R h o^{-/-}, P D E 6 \beta^{r d 1 / r d l}$ recipients were similar to wild-type. Maturation of the $\mathrm{Nrl}$-GFP+ rod precursors, including formation of spherule pre-synaptic structures, was observed in at least $65 \%$ of integrated rods in wildtype, Gnat $1^{-/-}$and $\mathrm{Crbl}^{r d 8 / r d 8}$ recipients, but was much lower in $\operatorname{Prph} 2^{+/ \Delta 307}, \operatorname{Prph} 2^{\text {rd2/rd2 }}$, $R h o^{-/-}, P D E 6 \beta^{r d l / r d l}$ recipients. When the rod precursors were transplanted at early, mid, and late stages of endogenous photoreceptor degeneration in the above models and wildtype, integration also varied, leading the investigators to conclude that $\mathrm{Nrl}-\mathrm{GFP}+$ rod integration was independent of outer nuclear layer thickness and rate of progression. In fact, this report confirmed a previous report ${ }^{10}$ that the intermediate filament, glial fibrillary acidic protein (GFAP), expressed by reactive glial astrocytes and Muller glia, and other gliosis/ scar-associated factors, such as chondroitin sulfate proteoglycans (CSPGs), are key regulators that limit integration across the different models. Furthermore the investigators noted that disruption of the outer nuclear layer, indicated by increased expression of the junctional proteins ZO-1 and Crb1, decreases integration of transplanted photoreceptors. Inhibiting these proteins, via siRNA disruption of ZO-1, and inducing enzymatic degradation of CSPGs, using chondroitinase $\mathrm{ABC}(\mathrm{ChABC})$, enhanced integration of $\mathrm{Nrl}$ $\mathrm{GFP}+$ rod precursors in the $\mathrm{Rho}^{-/-}$model, which exhibited the poorest integration rates, and highest degrees of gliosis and outer nuclear layer disruption. ${ }^{9,}{ }^{11-13}$ Together, these reports suggest that partial allelic rescue of mutant retinae harboring a variety of retinal degeneration-associated genes with Nrl-GFP+ rods depends on the degree of gliotic degeneration and outer nuclear integrity. Targeting these factors may allow improved integration despite varying genetic backgrounds.

Unipotent and differentiated retinal cells such as murine rod precursors and zebrafish cones and are not the only source of wild-type cells to restore structure and function in animal models of hereditary dystrophies. Since their isolation from mouse ${ }^{14}$ and human ${ }^{15}$ blastocysts, embryonic stem (ES) cells have emerged as a virtually unlimited source of differentiated cell types derived from all three (ectoderm, mesoderm, endoderm) germ layers. Advances in cell culture have enabled derivation of many specific retinal cells from ES cells, including retinal progenitors, ${ }^{16} \mathrm{RPE}^{17-20}$ and photoreceptors. ${ }^{21-24}$ Preliminary results following ES-derived RPE transplantation in Stargardt disease, a juvenile-onset form of macular degeneration most often caused by a mutation in $A B C A 4$, which encodes a cell surface pump that transports lipofuscin out of the RPE, has recently been reported. ${ }^{5}$ The report included two patients, one affected by Stargardt disease (and the other with non- 
exudative age related macular degeneration) who received allogeneic, subretinal ES-derived RPE transplants, with wild-type alleles of $A B C A 4$. Optical coherence tomography (OCT) showed that the ES-derived RPE integrated to the host RPE layer. Over four months, no tumors, epiretinal membrane, or immune rejection was detected. While an improvement in visual acuity was noted in the Stargardt patient, the phase I trial was not powered or intended to detect efficacy data. Nevertheless, this trial, the first for human ES cellderivative transplantation, offers a glimpse into the future of stem cell-derived retinal cell therapy for correction of hereditary retinal dystrophies.

While trials for blastocyst-derived ES cell retinal derivatives are taking place, clinical studies using another source of ES cell derivatives, so-called induced pluripotent stem (iPS) cells, are taking shape. ${ }^{25}$ Unlike traditional ES cells, iPS cells are transdifferentiated derivatives of isolable cells such as fibroblasts derived from skin, or from nucleated blood cells. ${ }^{26-29}$ Retroviral vectors encoding specific transcription factors, ${ }^{26,} 27$ non-integrating plasmids, ${ }^{30,31}$ synthetic modified mRNA, ${ }^{32}$ transduced recombinant proteins, ${ }^{33,} 34$ and small molecule epigenetic modifiers ${ }^{28,35}$ directly reprogram an adult cell into an embryonic cell, which can then be coaxed to retinal cell fates, ${ }^{20,36,37}$ using similar strategies as blastocyst-derived ES cells. Techniques allowing direct reprogramming of fibroblast to neurons ${ }^{38}$ and $\mathrm{RPE}^{39}$ — without requiring a potentially tumorigenic stem cell intermediate ${ }^{40}$ - have also been described. Direct reprogramming of easily isolable cells to differentiated retinal types, without stem cell intermediates, provide methods to understand mechanisms of transdifferentiation and cell type specification. However, for non-mitotic terminally differentiated cell types, such as photoreceptors and other retinal neurons, direct transdifferentiation may not provide enough cells for transplantation. Thus a transitamplifying stem cell intermediate may provide a method to generate the large number of cells required for grafting. For mitotic, terminally differentiated cells such as RPE, this technique may prove more useful, as transdifferentiated, mitotic RPE could be expanded in vitro to clinically significant numbers.

Cell therapy using donor rods and cones, their precursors, and RPE derived from fetal and stem cell sources allows simultaneous correction of the underlying genetic defect with wildtype alleles and replacement of degenerating, endogenous cells. This strategy has reached early-phase clinical trials, with ES-derived RPE for subretinal transplantation for Stargardt disease. ${ }^{5}$ Long-term safety and efficacy, at this time, remains uncertain but ongoing, larger clinical trials should provide greater clarity.

\section{GENE CORRECTION OF AUTOLOGOUS IPS CELLS FOR GENE AND CELL THERAPY}

While the vitreous and subretinal spaces are considered immunoprivileged sites, risks remain for immune rejection of the transplanted, allogeneic donor cells. ${ }^{41}$ Transplantation of autologous cells would theoretically reduce this risk. Autologous iPS cell-derived or directly transdifferentiated retinal cells can be generated from the patient's fibroblasts or blood. However, these somatic cells would carry the same genetic defect present in all cells of the affect patient. Thus transplantation of these autologous cells without correction of the underlying genetic defect would not be therapeutic. Emerging in vitro strategies however, 
may enable ex vivo gene correction followed by transplantation. Such an approach would have a three-fold purpose: to reduce risk of immune rejection via autologous cell therapy, to rescue the genetic defect, and to replace degenerating or promote endogenous cell survival.

The derivation of iPS and ES cells carries risks. This is because tremendous selective factors are involved in generating a viable cell line following prolonged cell culture. In the case of iPS cells, specific clones, which enjoy a survival advantage in culture because they have integrated crucial transcription factors (e.g. retroviral or episomal delivery of Sox2, Klf4, C$M y c, O c t 4)$, preferentially expand to outcompete other cells in cell culture. If this clone, or the number of viable clones, is relatively small, compared to the total population, a higher number of population doublings is required for the specific clone to dominate the cell line. Additional techniques, such as homologous recombination to correct a genetic mutation, also place selective pressure so that the genetically corrected cell line can be derived. The danger in these selective events lies in the accumulation of mutations, or mutational load, the resultant cell line will carry prior to transplantation of the cells into diseased retinae. A large mutational load may increase the risk of donor cell tumorigenesis. A recent report analyzed these phenomena following genetic correction of iPS cells derived from a patient with gyrate atrophy. ${ }^{42}$ In this case, iPS cells were derived from fibroblasts isolated from a patient with a homozygous point mutation in ornithine- $\delta$-aminotransferase (OAT) locus, the cause of gyrate atrophy (GA), a type of retinal degeneration thought to be related to primary RPE degeneration. Episomal reprogramming was employed to derive GA iPS cells free of transgene sequences. ${ }^{30}$ Homologous recombination using a BAC-based vector was used to correct the $O A T$ mutation. At different time points in culture, high density array comparative genomic hybridization $(\mathrm{aCGH})$ and exome sequencing were performed with the parental fibroblast line, iPS cells before and after gene targeting with homologous recombination, and after removal of the selection cassette (which allows selection of successfully gene targeted cells). While there was a significant mutational load in the iPS cell line, homology recombination and cassette removal contributed to relatively few additional changes. This study highlights that while genetic correction via homologous recombination adds little to the mutational load, and thus may be clinical useful, the mutational load acquired during iPS cell derivation may be substantial. Therefore prior to clinical transplantation of the gene corrected autologous, iPS retinal derivatives, the mutational load of the iPS cells must be carefully determined.

\section{SEQUENTIAL GENE AND CELL THERAPY}

Cell and gene therapy in hereditary retinal dystrophies need not be harnessed simultaneously through wild-type or ex vivo targeted alleles in donor transplanted retinal tissues. A strategy that offers sequential therapy, e.g. gene therapy of the donor or host cells, followed by cell transplantation, may also prove beneficial. A few recent reports describe this process.

One approach involves pre-treating the photoreceptor graft with anti-apoptotic therapy, such as the $\mathrm{X}$-linked inhibitor of apoptosis (XIAP), a caspase inhibitor. $\mathrm{Nrl}$-GFP+ rod precursors were pre-treated with adeno-associated virus (AAV-XIAP) prior to transplantation. Compared to non pre-treated donor cells, integrated AAV-XIAP pre-treated $\mathrm{Nrl}-\mathrm{GFP}+$ rod precursors demonstrated increased survival in the rd9 model, a mouse model of the most 
common cause of X-linked RP (XLRP), linked to mutations in the gene retinitis pigmentosa GTPase regulator (RPGR). No donor cell-specific retinal inflammation could be detected. ${ }^{43}$

In the reverse application, AAV-XIAP was injected at postnatal day 4 and 21, subretinally into the $P D E 6 \beta^{r d 10 / r d 10}$ mouse, which undergoes light-induced retinal degeneration. This allowed improved outer nuclear layer thickness and photoreceptor counts. In these experiments, adjunctive AAV-XIAP therapy was used to prolong survival of endogenous cells, and then these endogenous cells were again targeted with a variety of AAVs encoding PDE6 $\beta$. This strategy allowed prolonged targeting of endogenous photoreceptors with therapeutic gene vectors, as these cells survival longer because of the adjunctive antiapoptotic therapy. ${ }^{44}$ While this latter study is an example of sequential gene therapy without the cell therapy of the former study, ${ }^{43}$ one can imagine anti-apoptotic subretinal gene therapy to stimulate improved survival of endogenous photoreceptors, while donor photoreceptors are pre-treated with AAV-XIAP and then subsequently transplanted. Alternatively, adjunctive AAV-XIAP therapy of endogenous cells may prolong the window of opportunity for therapeutic cell transplantation therapy.

Another recent study highlighted the relative importance of rods and cones in human disease. While rods degenerate in many forms of RP, rod death may occur for many years without affecting central vision. This may result in nyctalopia but preserved visual acuity and activities of daily living in photopic conditions. However, when cones secondarily die later, central vision is lost, and the affected patient is markedly disabled. An exciting study demonstrated that genetic inactivation of $\mathrm{Nrl}$ in adult mice via a tamoxifen-inducible conditional, floxed allele, caused rods to be directly reprogrammed into cone-like cells, allowing preservation of photopic visual function. ${ }^{45}$ Inactivation of $\mathrm{Nrl}$ was associated with a conversion of rods to cells with a variety of cone-like histologic, molecular, and function features. Moreover, when $\mathrm{Nrl}$ was inactivated in the $\mathrm{Rho}^{-/-} \mathrm{RP}$ model, outer nuclear layer thickness was better preserved, as was the photopic b-wave. Therefore, rod reprogramming to cone-like cells through gene therapy ameliorates the effects of retinal degeneration. Taken together with earlier discussion, a sequential strategy may work in the following way: siRNA encoding $\mathrm{Nrl}$ inhibits endogenous $\mathrm{Nrl}$, allowing conversion of rods to cone-like cells, allowing slowed retinal degeneration and function, and an increased therapeutic window to transplant embryonic Crx-expressing cone precursors, ${ }^{8}$ to replace endogenous cones, at the time they begin to die despite gene therapy.

\section{ENCAPSULATED CELL TECHNOLOGY FOR PROTEIN THERAPY OF GENETIC DISEASE}

Intraocular encapsulated cell technology (ECT) has shown promise in geographic atrophy (GA), an advanced form of non-exudative age related macular degeneration (ARMD). ${ }^{46}$ Over time, as RPE die, large areas of choroidal tissue and vasculature become visible on physical examination. This advanced stage is known as GA and is associated with marked central vision loss because of death of the overlying macular photoreceptors. In a recent trial, patients with GA received ECT implants anchored to the scleral in an anterior location in the vitreous cavity. The interior of the ECT implant is filled with transfected cells that secrete ciliary body neurotrophic factor (CNTF) through a semipermeable capsule that 
houses the cells. Consistent levels of CNTF secretion persist for one to two years, and were associated with thicker outer nuclear layers, suggesting a decrease in photoreceptor death. The phase 2 trial showed that a subset of patients with baseline visual acuity of 20/63 or better who received the high-dose ( $20 \mathrm{ng}$ /day) CNTF secreting ECT implant, followed over one year, had a slower deterioration of visual acuity than those who received low-dose (5 ng/day) or sham surgery. Overall, the implant was well-tolerated.

While most associated with non-genetic forms of ARMD, GA can also be seen in Stargardt disease. ${ }^{47}$ It is possible that in the future, smaller ECT implants, anchored, free-floating, or subretinal implants could secrete specially packaged proteins that are otherwise dysfunctional in photoreceptors, other retinal neurons (e.g. bipolar cells in CSNB), or RPE (e.g. some forms of Leber's congenital amaurosis) associated with hereditary retinal dystrophies. It has been recently demonstrated ${ }^{34}$ that reprogramming proteins fused to a cell penetrating peptide (CPP), which contain a high proportion of basic amino acids (e.g. arginine and lysine) and are found in specific cell surface penetrating HIV peptides, ${ }^{48}$ allow transmembrane protein transduction to generate iPS cells from fibroblasts without viral delivery, genetic manipulation, or chemical treatments. In a similar way, ECT implants may one day rescue gene defects via secretion of corrective CPP-conjugated proteins to affected cell types, such as RPE65, GNAT1 and other protein products.

\section{CONCLUSION}

Cell and gene therapy offer promising strategies to simultaneously or sequentially correct gene deficiency and replace or preserve endogenous cells in hereditary retinal dystrophies. Sources of cells include primary fetal retinal cells, ES and iPS cell derivatives, as well as exogenous and endogenous directly reprogrammed cells. Current clinical trials make use of allogeneic, wild-type ES-derived (RPE) cell transplantation to correct the underlying dystrophy, such as Stargardt disease. However, the lack of immunogenicity of autologous cells like iPS-derived tissues potentially make them a more attractive, albeit much more expensive, population for clinically therapy, provided there is ex vivo gene correction. While iPS cell-derived RPE transplantation will see their clinical debut in trials in 2013 or $2014^{25}$ for the most common forms of non-exudative AMD and GA, their use to correct a hereditary retinal dystrophy has not yet been proposed. Nor has a trial been discussed using sequential gene and cell therapy, or ECT implants secreting CPP-conjugated peptides for therapeutic protein transduction. However, the clinical translation of these approaches remains a matter of time.

\section{References}

1. Bainbridge JW, Smith AJ, Barker SS, et al. Effect of gene therapy on visual function in Leber's congenital amaurosis. N Engl J Med. 2008; 358:2231-2239. [PubMed: 18441371]

2. Maguire AM, Simonelli F, Pierce EA, et al. Safety and efficacy of gene transfer for Leber's congenital amaurosis. N Engl J Med. 2008; 358:2240-2248. [PubMed: 18441370]

3. Cideciyan AV, Jacobson SG, Beltran WA, et al. Human retinal gene therapy for Leber congenital amaurosis shows advancing retinal degeneration despite enduring visual improvement. Proc Natl Acad Sci U S A. 2013; 110:E517-525. [PubMed: 23341635]

4. Streilein JW. Ocular immune privilege: therapeutic opportunities from an experiment of nature. Nat Rev Immunol. 2003; 3:879-889. [PubMed: 14668804] 
5. Schwartz SD, Hubschman JP, Heilwell G, et al. Embryonic stem cell trials for macular degeneration: a preliminary report. Lancet. 2012; 379:713-720. [PubMed: 22281388]

6. Lewis A, Williams $\mathrm{P}$, Lawrence $\mathrm{O}$, et al. Wild-type cone photoreceptors persist despite neighboring mutant cone degeneration. J Neurosci. 2010; 30:382-389. [PubMed: 20053919]

7. Pearson RA, Barber AC, Rizzi M, et al. Restoration of vision after transplantation of photoreceptors. Nature. 2012; 485:99-103. [PubMed: 22522934]

8. Lakowski J, Baron M, Bainbridge J, et al. Cone and rod photoreceptor transplantation in models of the childhood retinopathy Leber congenital amaurosis using flow-sorted Crx-positive donor cells. Hum Mol Genet. 2010; 19:4545-4559. [PubMed: 20858907]

9. Barber AC, Hippert C, Duran Y, et al. Repair of the degenerate retina by photoreceptor transplantation. Proc Natl Acad Sci U S A. 2013; 110:354-359. [PubMed: 23248312]

10. Kinouchi R, Takeda M, Yang L, et al. Robust neural integration from retinal transplants in mice deficient in GFAP and vimentin. Nat Neurosci. 2003; 6:863-868. [PubMed: 12845328]

11. Ma J, Kabiel M, Tucker BA, et al. Combining chondroitinase ABC and growth factors promotes the integration of murine retinal progenitor cells transplanted into Rho(-/-) mice. Mol Vis. 2011; 17:1759-1770. [PubMed: 21750603]

12. Singhal S, Lawrence JM, Bhatia B, et al. Chondroitin sulfate proteoglycans and microglia prevent migration and integration of grafted Muller stem cells into degenerating retina. Stem Cells. 2008; 26:1074-1082. [PubMed: 18218817]

13. Pearson RA, Barber AC, West EL, et al. Targeted disruption of outer limiting membrane junctional proteins (Crb1 and ZO-1) increases integration of transplanted photoreceptor precursors into the adult wild-type and degenerating retina. Cell Transplant. 2010; 19:487-503. [PubMed: 20089206]

14. Martin GR. Isolation of a pluripotent cell line from early mouse embryos cultured in medium conditioned by teratocarcinoma stem cells. Proc Natl Acad Sci U S A. 1981; 78:7634-7638. [PubMed: 6950406]

15. Thomson JA, Itskovitz-Eldor J, Shapiro SS, et al. Embryonic stem cell lines derived from human blastocysts. Science. 1998; 282:1145-1147. [PubMed: 9804556]

16. Ikeda H, Osakada F, Watanabe K, et al. Generation of $\mathrm{Rx}+/ \mathrm{Pax} 6+$ neural retinal precursors from embryonic stem cells. Proc Natl Acad Sci U S A. 2005; 102:11331-11336. [PubMed: 16076961]

17. Idelson M, Alper R, Obolensky A, et al. Directed differentiation of human embryonic stem cells into functional retinal pigment epithelium cells. Cell Stem Cell. 2009; 5:396-408. [PubMed: 19796620]

18. Maruotti J, Wahlin K, Gorrell D, et al. A simple and scalable process for the differentiation of retinal pigment epithelium from human pluripotent stem cells. Stem Cells Transl Med. 2013; 2:341-354. [PubMed: 23585288]

19. Buchholz DE, Pennington BO, Croze RH, et al. Rapid and efficient directed differentiation of human pluripotent stem cells into retinal pigmented epithelium. Stem Cells Transl Med. 2013; 2:384-393. [PubMed: 23599499]

20. Meyer JS, Shearer RL, Capowski EE, et al. Modeling early retinal development with human embryonic and induced pluripotent stem cells. Proc Natl Acad Sci U S A. 2009; 106:1669816703. [PubMed: 19706890]

21. Osakada F, Ikeda H, Sasai Y, et al. Stepwise differentiation of pluripotent stem cells into retinal cells. Nat Protoc. 2009; 4:811-824. [PubMed: 19444239]

22. Gonzalez-Cordero A, West EL, Pearson RA, et al. Photoreceptor precursors derived from threedimensional embryonic stem cell cultures integrate and mature within adult degenerate retina. Nat Biotechnol. 2013

23. Sasai Y. Next-generation regenerative medicine: organogenesis from stem cells in $3 \mathrm{D}$ culture. Cell Stem Cell. 2013; 12:520-530. [PubMed: 23642363]

24. Nakano T, Ando S, Takata N, et al. Self-formation of optic cups and storable stratified neural retina from human ESCs. Cell Stem Cell. 2012; 10:771-785. [PubMed: 22704518]

25. Cyranoski D. Stem cells cruise to clinic. Nature. 2013; 494:413. [PubMed: 23446394]

26. Takahashi K, Yamanaka S. Induction of pluripotent stem cells from mouse embryonic and adult fibroblast cultures by defined factors. Cell. 2006; 126:663-676. [PubMed: 16904174] 
27. Takahashi K, Tanabe K, Ohnuki M, et al. Induction of pluripotent stem cells from adult human fibroblasts by defined factors. Cell. 2007; 131:861-872. [PubMed: 18035408]

28. Shi Y, Desponts C, Do JT, et al. Induction of pluripotent stem cells from mouse embryonic fibroblasts by Oct4 and Klf4 with small-molecule compounds. Cell Stem Cell. 2008; 3:568-574. [PubMed: 18983970]

29. Loh YH, Agarwal S, Park IH, et al. Generation of induced pluripotent stem cells from human blood. Blood. 2009; 113:5476-5479. [PubMed: 19299331]

30. Yu J, Hu K, Smuga-Otto K, et al. Human induced pluripotent stem cells free of vector and transgene sequences. Science. 2009; 324:797-801. [PubMed: 19325077]

31. Jia F, Wilson KD, Sun N, et al. A nonviral minicircle vector for deriving human iPS cells. Nat Methods. 2010; 7:197-199. [PubMed: 20139967]

32. Warren L, Manos PD, Ahfeldt T, et al. Highly efficient reprogramming to pluripotency and directed differentiation of human cells with synthetic modified mRNA. Cell Stem Cell. 2010; 7:618-630. [PubMed: 20888316]

33. Zhou H, Wu S, Joo JY, et al. Generation of induced pluripotent stem cells using recombinant proteins. Cell Stem Cell. 2009; 4:381-384. [PubMed: 19398399]

34. Kim D, Kim CH, Moon JI, et al. Generation of human induced pluripotent stem cells by direct delivery of reprogramming proteins. Cell Stem Cell. 2009; 4:472-476. [PubMed: 19481515]

35. Hou P, Li Y, Zhang X, et al. Pluripotent Stem Cells Induced from Mouse Somatic Cells by SmallMolecule Compounds. Science. 2013

36. Homma K, Okamoto S, Mandai M, et al. Developing rods transplanted into the degenerating retina of Crx-knockout mice exhibit neural activity similar to native photoreceptors. Stem Cells. 2013; 31:1149-1159. [PubMed: 23495178]

37. Parameswaran S, Balasubramanian S, Babai N, et al. Induced pluripotent stem cells generate both retinal ganglion cells and photoreceptors: therapeutic implications in degenerative changes in glaucoma and age-related macular degeneration. Stem Cells. 2010; 28:695-703. [PubMed: 20166150]

38. Liu ML, Zang T, Zou Y, et al. Small molecules enable neurogenin 2 to efficiently convert human fibroblasts into cholinergic neurons. Nat Commun. 2013; 4:2183. [PubMed: 23873306]

39. Zhang K, Liu GH, Yi F, et al. Direct conversion of human fibroblasts into retinal pigment epithelium-like cells by defined factors. Protein Cell. 2013

40. MacLaren RE, Pearson RA, MacNeil A, et al. Retinal repair by transplantation of photoreceptor precursors. Nature. 2006; 444:203-207. [PubMed: 17093405]

41. Enzmann V, Faude F, Wiedemann P, et al. The local and systemic secretion of the proinflammatory cytokine interleukin- 6 after transplantation of retinal pigment epithelium cells in a rabbit model. Curr Eye Res. 2000; 21:530-534. [PubMed: 11035532]

42. Howden SE, Gore A, Li Z, et al. Genetic correction and analysis of induced pluripotent stem cells from a patient with gyrate atrophy. Proc Natl Acad Sci U S A. 2011; 108:6537-6542. [PubMed: 21464322]

43. Yao J, Feathers KL, Khanna H, et al. XIAP therapy increases survival of transplanted rod precursors in a degenerating host retina. Invest Ophthalmol Vis Sci. 2011; 52:1567-1572. [PubMed: 20926819]

44. Yao J, Jia L, Khan N, et al. Caspase inhibition with XIAP as an adjunct to AAV vector genereplacement therapy: improving efficacy and prolonging the treatment window. PLoS One. 2012; 7:e37197. [PubMed: 22615940]

45. Montana CL, Kolesnikov AV, Shen SQ, et al. Reprogramming of adult rod photoreceptors prevents retinal degeneration. Proc Natl Acad Sci U S A. 2013; 110:1732-1737. [PubMed: 23319618]

46. Zhang K, Hopkins JJ, Heier JS, et al. Ciliary neurotrophic factor delivered by encapsulated cell intraocular implants for treatment of geographic atrophy in age-related macular degeneration. Proc Natl Acad Sci U S A. 2011; 108:6241-6245. [PubMed: 21444807]

47. Fritsche LG, Fleckenstein M, Fiebig BS, et al. A subgroup of age-related macular degeneration is associated with mono-allelic sequence variants in the ABCA4 gene. Invest Ophthalmol Vis Sci. 2012; 53:2112-2118. [PubMed: 22427542] 
48. Frankel AD, Pabo CO. Cellular uptake of the tat protein from human immunodeficiency virus. Cell. 1988; 55:1189-1193. [PubMed: 2849510] 


\section{Homologous Recombination to Correct Gene Defects Prior to Autologous Stem Cell Derived Retinal Therapy}

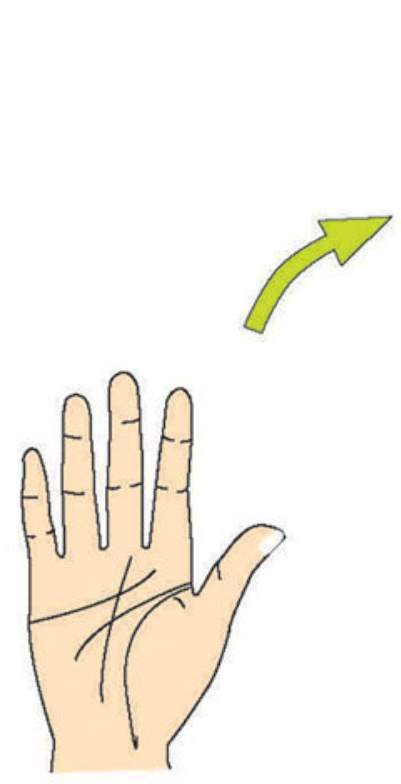

Patient with GA (mutation in Ornithine Delta Aminotransferase)

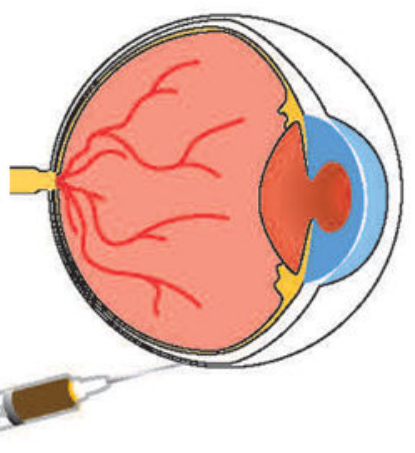

Subretinal transplantation of autologous, corrected iPSC derived RPE into original GA patient
Episomal Vectors

encoding reprogramming transcription factors

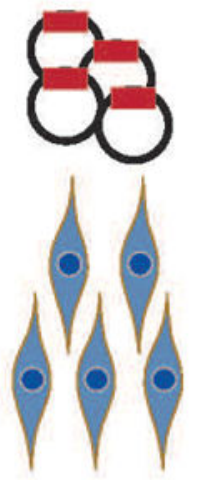

Fibroblasts isolated from skin of GA patient

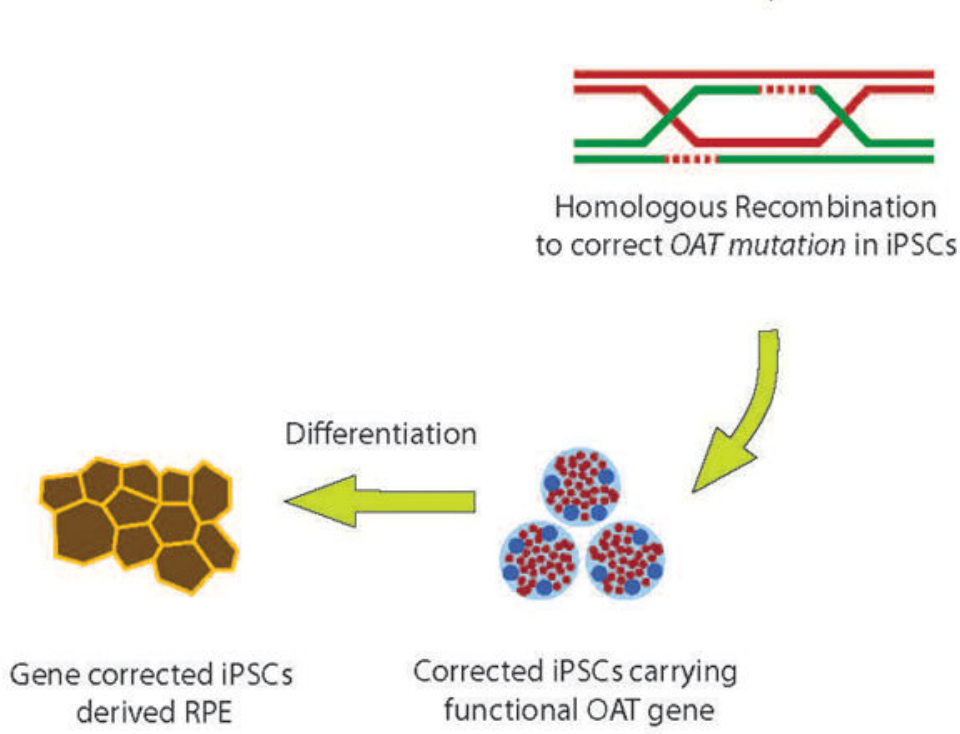

Figure 1. 
Sequential Gene and Cell Therapy

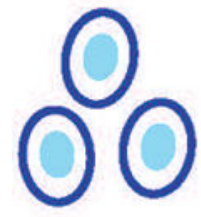

ESCS or iPSCs
Differentiation

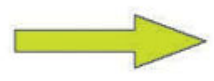

AAV-XIAP

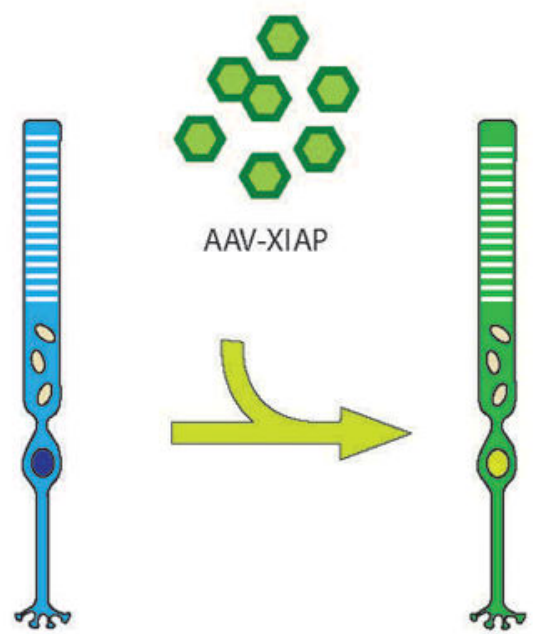

PRs

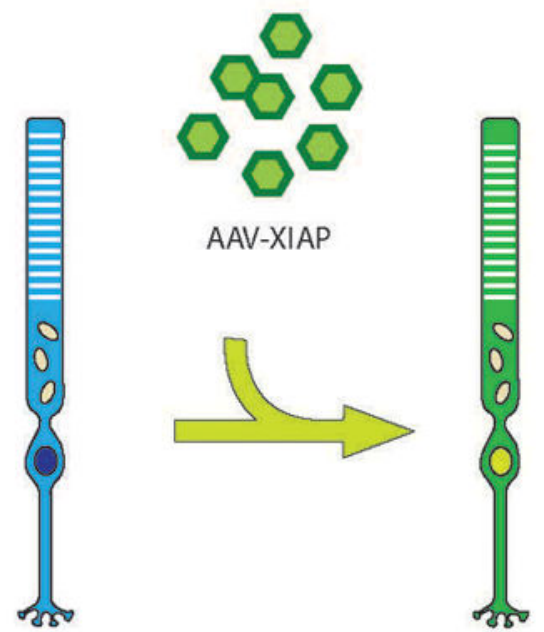

Transduced PRs

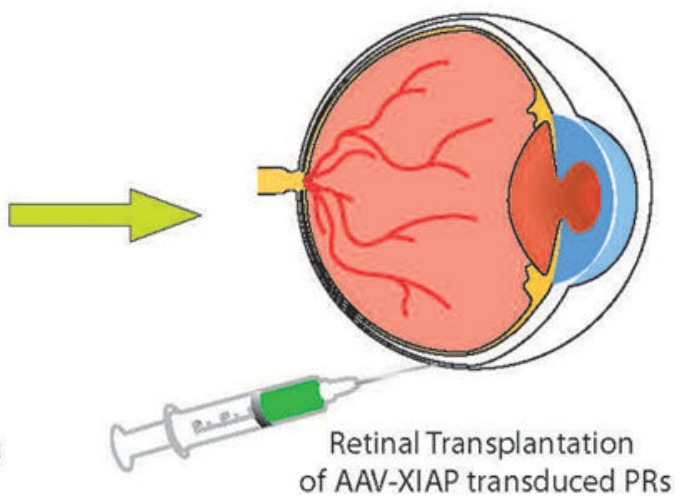

Figure 2.

Dev Ophthalmol. Author manuscript; available in PMC 2014 November 13. 


\section{Photoreceptor Reprogramming}
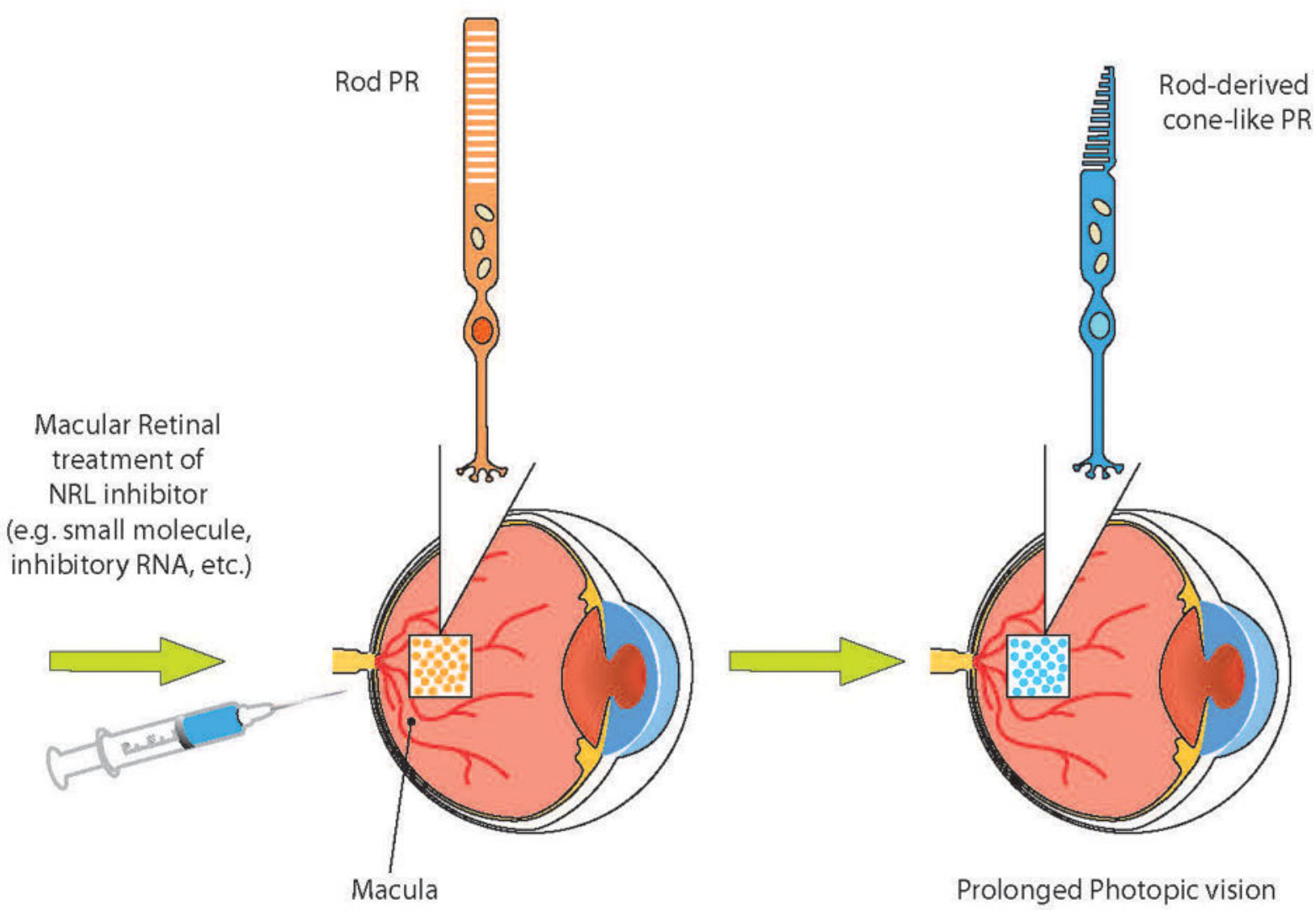

Figure 3. 